\title{
Über eine Reaktion des Amylalkohols.
}

\section{Von}

H. v. Wyss, E. Herzfeld und O. Rewidzoff.

(Aus dem chemischen Laboratorium der medizinischen Klinik in Zürich.)

(Der Redaktion zugegangen am 30. Januar 1910.)

Beschäftigt mit der Untersuchung der Körperflüssigkeiten auf oxydierende Fermente fanden wir, daß jeder normale Harn versetzt mit $\alpha$-Naphthol, p-Phenylendiamin, Sodalösung und $\mathrm{H}_{2} \mathrm{O}_{2}$ in bestimmter Weise intensiv blaue Farbe annimmt. Die dazu notwendigen Lösungen wurden folgendermaßen hergestellt:

1. 4,5 g $\alpha$-Naphthol kalt gelöst in $100 \mathrm{ccm} 50 \%$ oigem Alkohol.

2. 4,5 g p-Phenylendiamin kalt gelöst in $100 \mathrm{ccm}$ absolutem Alkohol.

3. $4,5 \mathrm{~g} \mathrm{Na}_{2} \mathrm{CO}_{3}$ gelöst in $100 \mathrm{ccm} \mathrm{H}_{2} \mathrm{O}$.

4. 3\% \% ige $\mathrm{H}_{2} \mathrm{O}_{2}$-Lösung.

Wenn man von den unter 1), 2) u. 3) angegebenen Jösungen je 4 Tropfen und von der Lösung 4) 10 Tropfen nimmt, tritt obige Reaktion ein. Wir versuchten, den betreffenden Körper aus dem Harn mit den verschiedensten Extraktionsmitteln zu extrahieren, und fanden dabei die auffallende Tatsache, daß Amylalkohol, mit obigen Körpern exkl. $\mathrm{H}_{2} \mathrm{O}_{2}$ versetzt, eine intensiv blaue Farbenreaktion zustande kommen läßt. Die genauen Bedingungen der Reaktion sind folgende:

$2 \mathrm{ccm}$ Amylalkohol,

4 Tropfen Lösung 1

4 Tropfen Lösung 2$\}$

4 Tropfen Lösung 3.

Es tritt rasch eine intensiv dunkelblau-violette Farbe auf, ohne daß man schüttelt, und auch wenn man den Luftzutritt durch Abschluß mit Xylol verhindert. Vorderhand verzichten wir 
480 H. v. Wyss, E. Herzfeld und 0. Rewidzoff, Über Amylalkohol.

darauf, eine Erklärung der Reaktion zu geben. Dagegen fügen wir hinzu, daß diese Reaktion schwach positiv noch bei folgenden Körpern ausfällt:

Isobutylalkohol (Wirkung ungefähr $4 \mathrm{mal}$ so schwach wie bei Amylalkohol, jedoch stärker wie bei folgenden)

Heptylalkohol schwach

Äthylalkohol »
Cetylalkohol
Glycerin
Wachs

Die vier letzten Körper zeigen nach etwa 5 Minuten eine schwache violette Färbung. Alle Reaktionen wurden zugleich mit einer Kontrollprobe angestellt, d. h. zu etwa $2 \mathrm{ccm} \mathrm{H}_{2} \mathrm{O}$ wurden je 4 Tropfen der obigen Lösungen exkl. $\mathrm{H}_{2} \mathrm{O}_{2}$ gegeben.

Bei folgenden Substanzen: Methylalkohol, Propylalkohol, Rohrzucker, Inosit, Methylacetat, Äther acetic., Aceton, Petroläther, Benzol, Toluol, Xylol, Chloroform, Fettsäuren, Harnsäure, Purinbasen, Kreatinin, Xanthin und einer Reihe anderer Körper fällt die Reaktion negativ aus. Selbstverständlich wurden Amylalkohol, Isobutylalkohol und einige andere positiv reagierende Körper auf Reinheit geprüft, da wir zuerst den Eintritt der Reaktion der Unreinheit der untersuchten Körper zuschrieben. Weitere Versuche sind noch im Gange.

Das Auffallende an der Reaktion ist also, daß bei der Bildung des Indophenols der Amylalkohol die Stelle einer Peroxydase plus $\mathrm{H}_{2} \mathrm{O}_{2}$ einnimmt. 